\section{Corona vaccine}

The most wanted thing at this moment throughout the globe is an effective vaccine against COVID-19. As Louis Pasteur, Edward Jenner paved the way of vaccines, corona vaccine has been introduced recently. It is thought that $60-70 \%$ of the global population must be immune to stop the virus spreading easily (herd immunity), so billions of people need to be vaccinated, even if the vaccine works perfectly, it's long way to go still.

\section{The development cycle of a vaccine from lab to clinic Pre-clinical testing}

Scientists test a new vaccine on cells and then give it to animals. So far, 86 vaccines are in pre-clinical phase of development.

\section{Phase 1 safety trial}

Scientists give the vaccine to a small number of people to test safety and dosage as well as to confirm that it stimulates the immune system.

\section{Phase 2 expanded trials}

Scientists give the vaccine to hundreds of people; split into groups, such as children and the elderly, to see if the vaccine acts differently in them. These trials further test the vaccine's safety and ability to stimulate the immune system.

\section{Phase 3 safety trials}

Scientists give the vaccine to thousands of people and wait to see how many become infected, compared with volunteers who received a placebo. In June, the F.D.A. advised vaccine makers that they would want to see evidence that vaccines can protect at least 50 percent of those who receive it. In addition, phase 3 trials are large enough to reveal evidence of relatively rare side effects.

\section{Combined phases}

One way to accelerate vaccine development is to combine phases.

\section{Paused}

If investigators observe worrying symptoms in volunteers, they can put a trial on pause. After an investigation, the trial may resume or be abandoned.
(BIRDEM Med J 2021; 11(1): 1-5)

\section{Early or limited approval}

China and Russia have approved vaccines without waiting for the results of phase 3 trials. Experts say the rushed process has serious risks.

\section{Approval}

Regulators in each country review the trial results and decide whether to approve the vaccine or not. During a pandemic, a vaccine may receive emergency use authorization (EUA) before getting formal approval. After the regulatory review, vaccines go into manufacturing. Even after being a vaccine is licensed, researchers continue to monitor people who receive it to make sure it is safe and effective.

\section{Corona vaccine evolution}

Phase 1: 41, Phase 2: 17, Phase 3: 13, Approved: 3-5 already approved for full use.

Vaccines typically require years of research and testing before reaching the clinic, but scientists are racing to produce a safe and effective coronavirus vaccine by next year. The first vaccine safety trials in humans started in March 2019 and now 13 have reached the final stages of testing. This time is with new technology with genetic vaccine.

\section{Vaccine research}

Genetic vaccine: Vaccine that deliver one or more of the coronavirus's own genes into our cells to provide an immune response - about 10 gene based vaccine in pipeline from different countries of the world. Most advanced stage is Moderna (USA) mRNA vaccine and Pfizer-Biontech (US \& German) mRNA vaccine. They have completed phase three trial .mRNA vaccine is the most recent concept,it was not done before.mRNA is injected into body with fat globule as vehicle. mRNA is vulnerable to degradation at room temperature.

Viral vector vaccine: also genetic vaccine; Virus has been engineered to carry corona genes. This viruses are genetically modified, they do not produce disease by themselves.; they shuttle the genetic material. Some vector gene enter the body to produce viral spike protein, 
stimulate antibody production. There are 14; eminent three are sputnik V of Russia, one by AstraZeneca oxford university and that of Johnson and Johnson.

Protein based vaccine: Vaccine that contain corona virus proteins but no genetic material, some vaccine contain whole protein, some fragments of them. Some pack many of these molecules on nanoparticles. There are about 22 companies throughout the glove in their trial of tomorrow's vaccine.

Inactivated or attenuated coronavirus vaccines: Vaccines created from weakened coronaviruses or coronaviruses that have been killed with chemicals. There are 10 vaccines of this kinds. Sinopharm the state owned and sinovac the private Chinese company is far ahead of other trials of these vaccines. These are well tested method of vaccine production since long ago.Vaccine development takes longer time but renders robust immunity to recipient. These vaccines have been approved for emergency use in UAE and many other Arab country and and mainland China .Many peoples has been vaccinated there with claimed demonstrated successes.

Repurposed vaccine: As the name says, here vaccine already in use for other diseases that may protect against COVID-19. Repurposed vaccine in fact is not counted in this period for fight against corona. Many institutes including the Murdoch children research institute, Australia, Serum institute of India are in this effort.

\section{Profile of vaccine in field 1. Pfizer BioNTech Vaccine}

On November 9, New York-based Pfizer and the German company BioNTech made history by presenting preliminary data indicating that their coronavirus vaccine was over 90 percent effective.. A week later, Moderna reported similar findings with a similar vaccine.

Vaccine caused volunteers to produce antibodies against SARS-CoV-2, as well as immune cells called T cells that respond to the virus.It is given by intramascular injection.It is an RNA vaccine composed of neucleosidemodifiedmRNA(modRNA) encoding a mutated form of the spike protein of SARS-CoV-2, which is encapsulated in lipid nanoparticles. Vaccination requires two doses three weeks apart. one taking first dose must take the 2nd dose with that of Pfizer BioNTech Vaccine not others. On November 8, Pfizer released preliminary analysis of the first 94 cases. Ten days later, they followed up with the final results, based on 164 cases. Pfizer and BioNTech concluded that the vaccine is 95 percent effective. Among people over 65, who often have weak responses to vaccines, it was 94 percent effective. On December 2, the United Kingdom gave emergency authorization to Pfizer and BioNTech's vaccine. FDA approved EUA on the $10^{\text {th }}$ December..They.did not study whether vaccine could stop transmission.It's ability to prevent severe infection in children,pregnant woman, immunocompromised people and it's duration immune effect are currently unknown.

Pfizer and BioNTech expect to manufacture over 1.3 billion doses of their vaccine worldwide by the end of 2021. The vaccine has been designed and developed by BioNtech and manufactured and distributed by Pfizer and Fosun pharmaceuticals. On 8 December, 90-yearold Margaret Keenan became the first patient to receive the vaccine at University Hospital in Coventry, with 800,000 more doses expected to be given in the coming weeks.Up to four million people may now receive the vaccine before the end of the month.It has been introduced in Canada and USA also.

Storage difficulty: Vaccine to be taken to destination country in dry ice pack; each pack holding 5000 doses. The destination country can choose to store in freezer farm up to six months in minus 70 degree centigrade. In the opened dry ice pack vaccine has ten days to reach the vaccination centre. Once delivered to the Centre vaccine can be stored up to 5 days in fridge between 28 degree centigrade..

\section{Moderna vaccine}

On November 30, the Boston-based company Moderna announced it was applying to the Food and Drug Administration for an emergency use authorization, less than a year after it launched the first clinical trial for a coronavirus vaccineIf Moderna receives the authorization, the first injections of its vaccine could start on December 21.

In January 2020 they began developing a vaccine for the coronavirus. The vaccine contains genetic instructions, when injected into cells, the messenger RNA co-opts the machinery used to make proteins to build tiny replicas of the spike protein that studs the 
outside of the coronavirus. The immune learns to recognize and block the spikeThe researchers estimated that the vaccine had an efficacy rate of 94.1 percent, far higher than experts had expected when vaccine testing began. While it's not clear how long this efficacy will last, Moderna has found that after three months the trial participants still have a strong immune defense against the coronavirus...Moderna uses the same approach as the Pfizer vaccine. It is given in two intramascular doses, four weeks apart. It is easier to store than Pfizer's, because it stays stable at $-20 \mathrm{C}$ (fridge for ice cream temperature) for up to six months. They.did not study whether vaccine could stop transmission. It prevent disease not infection.It's ability to prevent severe infection in children,pregnant woman, immunocompromised people and it's duration immune effect are currently unknown

\section{Sputnik-V}

The Gamaleya Research Institute, part of Russia's Ministry of Health, has created a vaccine based on two adenoviruses. Preliminary results announced in November indicate that the vaccine has a high efficacy rate in Phase 3 trials. The Gamaleya National Center of Epidemiology and Microbiology is the world's leading research institution founded in 1891.

The center runs one of the unique "virus libraries" in the world and has its own vaccine production facility. Gamaleya produced the vaccine, initially called GamCovid-Vac, from adenoviruses called Ad5 and Ad26. Both kinds have been tested as vaccines over the years. By combining them, the Russian researchers hoped to avoid a situation in which the immune system could learn to recognize the vaccine as a foreign object that needed to be destroyed. They expect more immune response and more prolonged response by using two different vectors. Their study population was less .. Based on 20 cases of COVID-19 among the trial participants, Russian scientists estimated that the vaccine demonstrated 92 percent efficacy. As with the three other vaccines with announcements of Phase 3 efficacy AstraZeneca, Moderna, and Pfizer - the Gamaleya Research Institute has yet to publish their latest findings in a peer-reviewed scientific journal.It has no storage problem.Two doses 3 weeks apart is needed.India has prebooked it along with 4 other vaccines(not moderna,not Pfizer-BioNTech).It is dry (lyophilized) vaccine can be stored between +2 to +8 degree Celsius,so easy to distribute.

\section{Adenoviral vaccines}

Adenoviral vectors are considered extremely safe and are some of the easiest to engineer. Vectors are viruses that have had their gene responsible for replication removed. Therefore, they no longer pose any threat of infection. The engineered virus, called adenovirus are designed to shuttle a gene with the code of a coronavirus $\mathrm{S}$ (spike) protein from SARS-CoV2 into our bodies where our cells will read. This inserted component is safe for the human body. This self-made spike protein would train our bodies to detect and terminate any real SARS-CoV2 before the covid-19 wreaks the havoc. The technique has been in development for more than 3 decades.CanSino biologic(china), Johnson\&Johnson (USA), the oxford university and Gamaleya centre is using this technology. Adenoviruses, which are found in adenoids and regularly cause acute respiratory infections, have become the viruses that are most commonly used to engineer vectors.. Scientists from the Gamaleya Center have been working on adenoviral vector-based vaccines since the 1980 s, and now lead the world in developing these types of vaccines Gamaleya Center,only uses the two-vector platform .With Viral vector vaccine first dose can be boosterd by the $2^{\text {nd }}$ dose with other viral vector vaccine. Astrzeneciaoxford is now going a trial of second dose with sputnik$\mathrm{V}$;it is not possible with mRNA vaccines.

\section{Johnson \& Johnson vaccine}

Janseen the pharmaceutical wing of Johnson \& Johnson developed vaccines for Ebola and other diseases with Ad26 and have now made one for the coronavirus levrages theirdVac@technology platform. They launched a Phase 3 trial with up to 60,000 participants in September. Unlike other Phase 3 trials, Johnson \& Johnson administered just a single dose instead of two. On Nov. 16, Johnson \& Johnson announced that they were launching a second Phase 3 trial to observe the effects of two doses of their vaccine, instead of just one. They have launched ENSEMBL 1 and ENSEMBLE 2 the two designed study to register clinical trial, as well as publishing results and making both clinical Study Reports and clinical trial participant data available for sharing with researchers through the Yale University Open Data Access (YODA) Project. 


\section{AstraZeneca \& University of Oxford Vaccine}

The British-Swedish company AstraZeneca and the University of Oxford developed a vaccine based on a chimpanzee adenovirus. On November 23, they announced that a preliminary analysis of their Phase 3 trial revealed the vaccine was up to 90 percent effective, depending on the dosage. The vaccine began Phase $2 / 3$ trials in the United Kingdom and India (where it's known as Covishield).

On November 19, researchers published the first findings from the Phase 2/3 trials in the United Kingdom. They looked in particular at how people at different ages responded to the vaccine, studying 160 people aged 18 to 55 years old, 160 between 56 and 69, and 24070 years or older. They did not observe any serious side effects at any age. Encouragingly, the older volunteers produced about as many antibodies against the coronavirus as the younger ones.

\section{Oxford University/AstraZeneca vaccine}

Trials of the Oxford vaccine show it stops $70 \%$ of people developing COVID symptoms. The data also shows a strong immune response in older people. There is also intriguing data that suggests perfecting the dose could increase protection up to $90 \%$. It is given in two doses 3 weeks apart.. It is made from a weakened version of a common cold virus from chimpanzees, that has been modified to not grow in humans. Scientists at AstraZeneca and the University of Oxford on Tuesday became the first vaccine developers to publish their full data in a peer-reviewed scientific journal, Lancet confirming earlier claims that the vaccine is 70 percent effective overall. The AstraZeneca-Oxford vaccine, however, can be stored long term at ordinary refrigerator temperatures, which could ease its distribution throughout the world.

Unlike Pfizer/BioNTech and Moderna's mRNA products, the AstraZeneca vaccine is cheap, can be stored at ordinary fridge temperatures, is easy to manufacture and presents the best hope at the moment for a vaccine for the billions rather than the few. The UK, the rest of Europe and Canada and India could approve it in the coming weeksThe coronavirus vaccine developed by the University of Oxford is highly effective at stopping people developing COVID-19 symptoms (prevent not only diseases but also infection).

\section{China vaccines}

China currently has five coronavirus candidates from four companies which have reached phase 3 clinical trials. Together, they have rolled out phase 3 trials in at least 16 countries. China National Biotec Group (CNBG), a unit of Sinopharm. The UAE approved emergency use for frontline workers in September. CNBG was capable of producing more than one billion doses in 2021.Compared with Pfizer and Moderna, most Chinese vaccines do not require freezing temperatures for storage, making transport and distribution much easier, especially in developing countries that lack cold storage capacities...

\section{Sinopharm Vaccine}

Sinopharm, meanwhile, uses a longstanding approach that has proven effect in other vaccines, such as polio and flu shots. Their coronavirus vaccines employ an inactivated whole virus to prompt the body to develop immunity and only need to be stored at standard refrigerator temperatures of 2 ÚC to 8 ÚC (36ÚF to 46ÚF). Other Chinese vaccines in production can be kept at similar temperatures. Coronavirus vaccine: China vaccine $86 \%$ effective, UAE says. Coronavir. 1 million Chinese injected with Sinopharm vaccine under emergency use scheme. '

\section{Sinovac Chinese vaccine}

Sinovac Biotech, a private Chinese company, developed an inactivated vaccine called CoronaVac. In July, Sinovac launched a Phase 3 trial in Brazil, followed by others in Indonesia and Turkey. While Sinovac has yet to release late-stage trial data.They have trial in many arab and other countries

\section{Bangladesh situation}

The government is not thinking about procuring the vaccines of Pfizer and Moderna for obvious reason.Sinovac was allowed to have their trial here.The technical set up is there but the company asked for Cofinancing which Bangladesh government did not agree.

The government has recently signed a memorandum of understanding (MoU) with the Serum Institute of India (SII) and Bangladesh's Beximco Pharmaceuticals to get three crore doses of the vaccine developed by Oxford University. Bangladesh will get this vaccine at the early stage (first part of January 2021) and it will be preserved at Beximco's cold storage. So, we shall be able to give 
this vaccine to our 1.5 crore population - two doses for each person. "

The Bangladeshi drug maker Globe biotech has ended a memorandum of understanding with international centre for diarrhoeal disease research,Bangladesh on trial of vaccine candidate BANCOVID the BANCOVID had recently been added to the WHO list of draft landscape of candidate vaccines.

\section{Who may get it first?}

National Immunization Technical Advisory Groups (NITAGs) has already set priorities for the distribution of vaccine doses once those will be found. Those who are working on the frontline in tackling the corona pandemic, including the health workers and law enforcers, will get the first priority. There is suggestion providing the media workers, elderly public representatives and elderly freedom fighters with the vaccine in the first phase. They also recommended giving the vaccine on a priority basis to the people having comorbidities and the senior citizens. India and UK governments have set of prioritization of vaccine seeker. NITAGs recommended providing the vaccine free of cost, but the government wants to take \$4-\$5 equivalent money for each dose of the vaccine. Country needs an urgent Vaccine policy.

\section{Complexity of vaccine introduction}

Getting the vaccine from the factory to people's arms could pose some major challenges. Like Moderna's vaccine, Pfizer and BioNTech's preparation is based on mRNA, poses problem with preservation and transport. Most vaccines (except those with inactivated virus vaccine)currently in trials, it requires two doses. Ensuring that peoples return after three weeks for their second injection will add an extra layer of complexity to the vaccine's distribution. Cost will definitely be a problem.

\section{Realities to realize}

Cost at present per dose: Sinopharm (China) \$145 for two shots. Sino vac (China) began selling $\$ 60$ for two shots. Oxford-AstraZenecea \$4 per dose, Pfizer BioTech $\$ 20$ per dose less than that of Moderna \$33 per dose. Sputnik V \$10 per dose.

Temperature factors: Regular fridge temperature for all except Moderna and Pfizer which need chilling subAntarchtic temperature.
Corona vaccine is possible. You need to take two doses, you start developing immunity 7 days after the last dose.

Job to immunize 6 billion people of the world is enormous.. With a fast-moving pandemic, no one is safe, unless everyone is safe. "Corona vaccine is my right" ,the equity programme is on the air. The COVAX facility forms a key part of the a ground-breaking global collaboration to accelerate the development, production and equitable access to COVID-19 tests, treatments and vaccines

At the same time, the six front running vaccine candidates have had a total of over US\$12 billion of tax payer and public money poured into them. Deals from pharmaceutical companies to develop COVID-19 vaccines are shrouded in secrecy, and details from them that are released reveal worrying terms It is prudent to urge governments - which have provided funding to these companies - to demand transparency on vaccine licensing deals and on trial costs and data

\section{Conclusion}

With a fast-moving pandemic, no one is safe, unless everyone is safe. Trials must show vaccines are safe. Huge-scale development must happen for the billions of potential doses. Regulators must approve the vaccine before it can be given. Researchers still need to find out how long any protection may last. Be aware that antivaccine stories are spread online through social media. These posts are not based on scientific advice (or blend facts with misinformation). The announcement gave us the headline, but there is a still lack of fine detail. Everyone is happy if a vaccine is both safe and effective. Checks continue after approval to make sure there are no further side effects or long-term risks. Natural immunity may not be long-lived and immunization could offer more protection.

Guidance says there are no safety concerns, vaccine to be given to people who suffered from COVID or with "long-COVID" either. If enough people are immune, then the virus would stop spreading and we would not need other measures. However, the manufacture and distribution of a vaccine will take some time.Moreover most Vaccine does not ensure stopping transmission(prevent disease not infection) So, testing, lockdowns, social distancing and mask wearing are going to be a feature of our lives for a while yet. 
